\title{
Practical applications of atomic force microscopy in biomedicine
}

\author{
Nicola GALVANETTO ${ }^{\mathrm{a}, \mathrm{b}^{*}}$ \\ ${ }^{a}$ International School for Advanced Studies (SISSA), via Bonomea 265, Trieste 34136, Italy. \\ ${ }^{b}$ Department of Biochemistry and Department of Physics, University of Zurich, 8057 Zurich, Switzerland. \\ ${ }^{*}$ Correspondence: nicola.galvanetto@sissa.it \\ https://doi.org/10.37175/stemedicine.v1i2.15
}

\begin{abstract}
The last thirty years of progress of atomic force microscopy (AFM) applied to living matter is reviewed with a focus on potential uses in drug discovery or screening of patient samples. AFM-based technologies are still at Proof of Concept level - or below, however, they are particularly promising for i) live imaging of unlabeled membrane proteins and ii) nanomechanical screening of biological samples, e.g. cancer biopsies.
\end{abstract}

Keywords: Atomic force microscopy · Cell membranes · Membrane proteins · Unroofing · Applications

\section{Background}

The progress of science is usually a graded process, but once in a while it shows discontinuities due to some disruptive revolutions. In physics, there have been mostly conceptual revolutions: theories that allow to link experimental results that were previously uncorrelated; in modern biology, on the contrary, there have been mostly technological revolutions: techniques that enable (previously) unthinkable experiments.

The invention of the Atomic Force Microscope (AFM) (1) (Figure 1) is one of these revolutions.

\section{AFM is an enabling technology still under development}

\subsection{AFM is a special kind of microscope}

The word microscope derives from the Greek words mikros - "small" - and skopein - "to look". If we took literally this definition, it wouldn't be right to call the AFM really a 'microscope', because AFMs are metaphorically much closer to touch than to sight. Indeed, the basic working principle of the AFM is a tip that touches and moves on top of a surface (Figure 1).

The working principle is simple, but the necessary technology to bring it to sub-nanometric precision is all but not trivial. Broadly speaking, the goal is to raster scan the surface of the sample with a tiny probe

Received: Nov 7, 2019; Accepted: Dec 29, 2019.

(c) The Author(s). 2019 This is an Open Access article distributed under the terms of the Creative Commons License (http://creativecommons.org/licenses/by/4.0/) which permits unrestricted use, distribution, and reproduction in any medium or format, provided the original work is properly cited. (cantilever tip), to record the scanned information as $\mathrm{X}-\mathrm{Y}-\mathrm{Z}$ coordinates, and to reconstruct this map into a topographical image true to the original sample. A modern AFM (2) is constituted by a micrometric cantilever with a sharp tip on one side, and connected to a cantilever holder on the other (Figure 1). The cantilever holder is fixed to a piezo tube (a piezoelectric crystal fused in a tubular volume) necessary to move the cantilever tip in the X-Y-Z directions. The piezo tube is a key element because it uses the special property of piezo crystals to deform in a very accurate manner under high electric voltages, thus to move the cantilever with sub-nanometric precision. The forces to which the cantilever is subjected are constantly monitored through a laser beam that points to the cantilever and reflects into a photodetector (Figure 1): in this way, even the smallest deflections of the cantilever are detected by the photodetector as movements of the laser spot. Moreover, in the regime of small deflections, the cantilever behaves like a spring, therefore the AFM can be used not only to raster scan a surface, but also to measure forces applied to an extremely small area (i.e. the apex of the tip). The power of the AFM comes from the fine intercombination of these elements and from the miniaturization that started in the $1960 \mathrm{~s}$, on the wave of the silicon revolution.

To summarize, an AFM is the combination of three advanced technologies: piezoelectric crystals, microfabrication for the cantilever tip, laser and electronics for the control device. In the following, the advancements of microfabrication and electronics that represent the domains of major gain of AFM performance of the last few years are briefly reviewed. 


\section{2 'Make it smaller and faster' (microfabrication \& electronics)}

The resolution of the AFM depends on the size of the probe that is used to scan the surface of interest. In order to follow nanometer features, tips with an apex with nanometer radius of curvature are essential. Moreover, if the sample presents densely packed hills and holes, the tip necessitates to enter the holes and follow every detail to generate a faithful topographical representation of the sample. For these reasons, scientists developed methods to grow on top of the cantilever - or on top of the cantilever tip itself (Figure 2a) - an additional ultra-sharp tip made of carbon atoms. This additional tip can be several $\mu \mathrm{m}$ high and can have a radius of curvature of 1-2 nm (3), in order to follow the finest details of the sample.

But it is not only a matter of tips, indeed the quality of the topographical reconstruction depends also on the size of the cantilever. As a general principle, the smaller the better, because a small lever is softer (less invasive) and lighter (less inertia). The challenges were a) to manufacture small and precise levers and b) to fit the AFM accordingly. The advancements in microelectronics allowed to have the right technology to build small probes, and these small probes were also modified (sculptured) with Focused Ion Beams to make them even softer and lighter (Figure 2b) (4). Once small probes were made, a standard AFM became inadequate, in particular in the laser-photodetector coupling. In order to focus the laser beam onto these new small probes, the AFMs were customized with an optical objective necessary to focus the light onto the smaller reflection area of the new levers.

Now that all the ingredients for better AFMs were ready, the last step was to make them faster. An AFM image used to require several minutes to be recorded. Therefore, AFMs were equipped with special ultra-fast electronics giving birth to what is now called High-speed AFM (HSAFM), which can be considered a second revolution in the field (5).

\subsection{AFM is used to generate images or to probe the nanomechanics}

There are two kinds of output that an AFM can generate: images or force curves.

AFM can be used to scan a surface, thus to record the surface topology of a sample (Figure 4a). These images can be collected in the so called 'contact mode' i.e. when the tip literally touches the surface moving laterally like in Figure 1; but the images can be generated also in the so called 'non-contact' mode or in 'intermittent contact' mode. In these cases, the tip, in addition to a lateral movement, is forced to oscillate in the vertical direction. These last modes of operation are particularly advantageous with soft samples, to prevent their damage.

But AFM is also a force probe, therefore the tip can also be used in one single location to probe the nanomechanical properties of the material. The tip is forced to move downwards and then upwards, while collecting the deflection of the cantilever. In this way it is possible to collect indentation curves or unfolding curves of a protein (Figure 6c).

In Section 3 I will present the applications in biomedicine of the AFM image generation, while in Section 4 and 5 I will discuss the use of AFM as a force probe of biological matter.

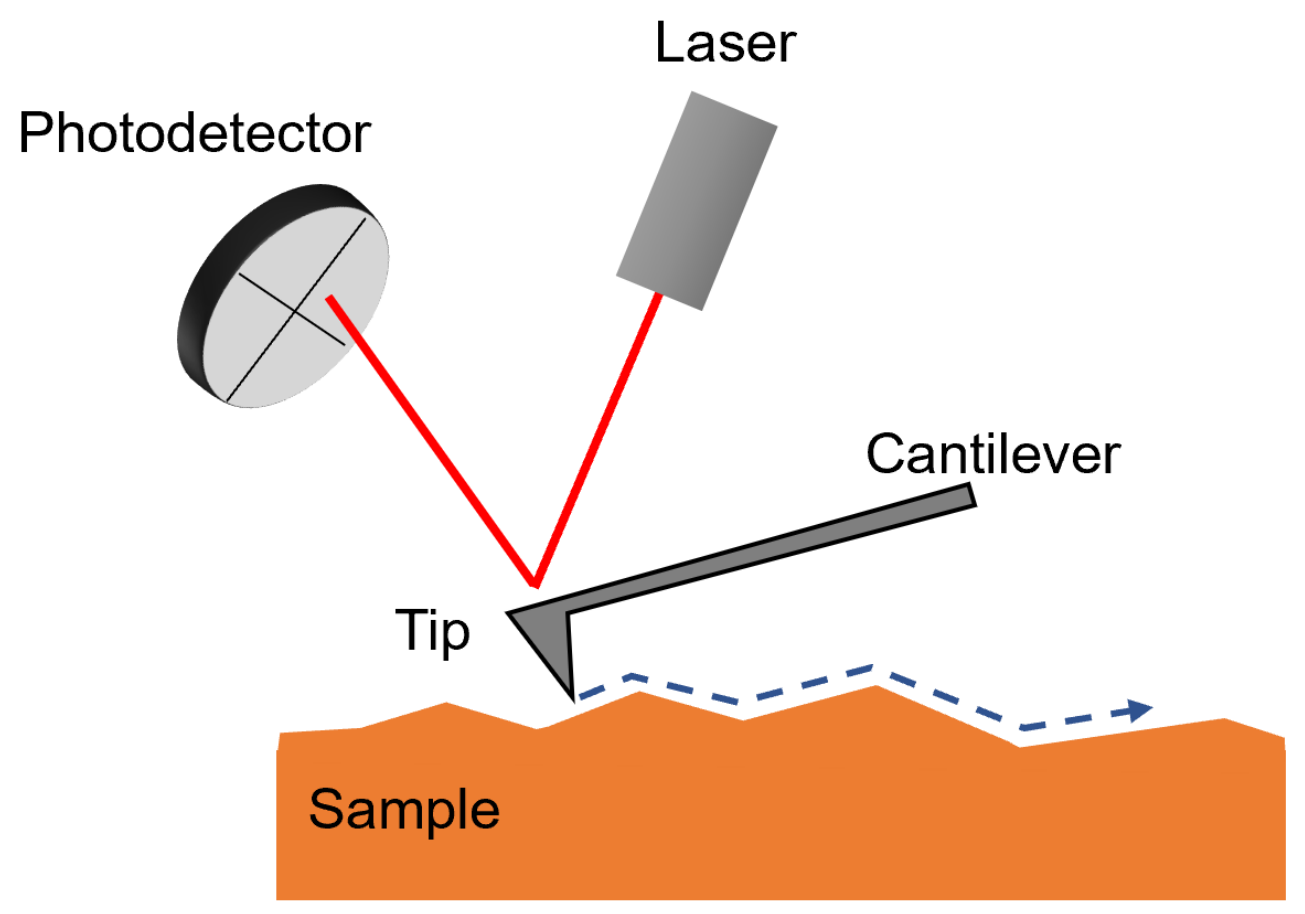

Figure 1. Sketch of an AFM probe moving on top of the imaged surface and basic scheme of an AFM device. 
a

Carbon nanocone on the tip

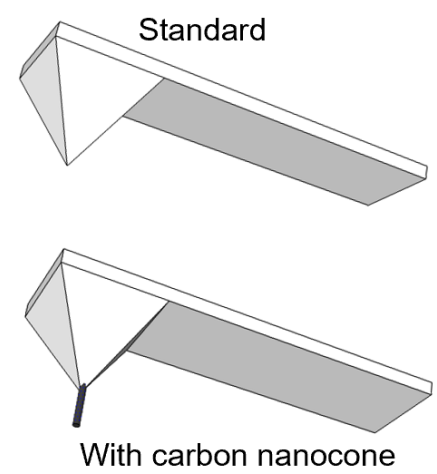

b

\section{Cantilever with special shapes}

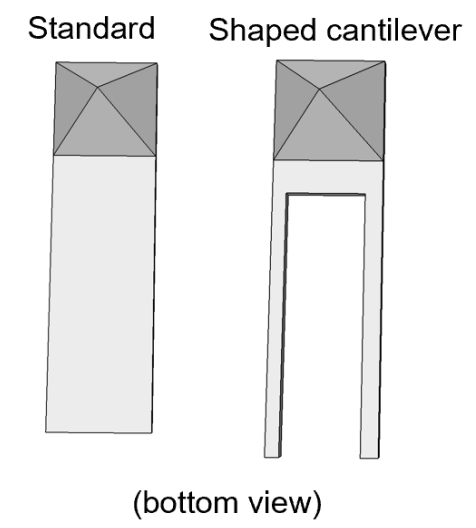

Figure 2. a: ultra-sharp tip (with carbon nanotube grown on top of a silicon tip; like in ref. (5)). b: bottom views of modified cantilevers for enhanced temporal resolution (like in ref. (4)).

\section{AFM is a microscope for unlabeled molecules}

Biology and modern medicine make extensive use of imaging techniques, and in particular of fluorescence microscopy $(6,7)$ (Figure 3c). The general idea underlying fluorescence microscopy is to obtain signals from the specific biomolecule of interest, and to associate it with its spatial distribution within the sample, sometimes also for quantitative assessments. The biomolecule of interest is in almost all cases not fluorescent - i.e. invisible - therefore it needs to be labeled with another fluorescent molecule to be detected. In any case, with the exception of Forster Resonance Energy Transfer, no structural information of the biomolecules can be obtained with fluorescence microscopy.

To obtain structural information, structural biology and pharmacology use X-ray crystallography, nuclear magnetic resonance, and more recently cryogenic electron microscopy (cryo-EM) to investigate the internal structure of the biomolecule of interest $(8,9)$ (Figure 3a). With these techniques it is possible to reach sub-nanometer resolution of the $3 \mathrm{D}$ arrangements of the amino acids in the case of proteins, which is very relevant in drug design. To gather this structural information there are some experimental steps which include the purification of the biomolecule, or its freezing, that necessarily does not allow to keep the molecules in a physiological (not even almost-physiological) environment.

There are situations, or scientific prerequisites, where either the label cannot be applied, or the project necessitate structural and dynamical information of the biomolecule in an almost-physiological environment. For these kinds of situations, the AFM is the ideal microscopy technique because it sits exactly in between fluorescence microscopy and crystallography/cryo-EM (Figure 3b).

\subsection{Purified proteins reconstituted in membranes}

Despite of being the target of about $50 \%$ of modern drugs (10), structural studies have been particularly complicated, with membrane proteins that are not prone to crystallization because they have a biphasic surface (hydrophilic at the poles and hydrophobic at the equator to accommodate the membrane), therefore it is difficult to measure the structural effect of a ligand.

In the late 1980s, the newborn AFM that was invented for applications on semiconductors or insulators also entered the biology departments, and the first pioneering studies on membrane proteins began. The first membrane protein for which it was developed a protocol for AFM imaging was bacteriorhodopsin (11), a membrane protein that form $2 \mathrm{D}$ crystals called purple membranes. Some years later it was possible to reach sub-molecular resolution and compare the AFM topographs with the atomic model derived from electron microscopy (12). The real AFM advantages over electron microscopy started to be evident at this point: indeed, AFM could be operated in liquid, i.e. emulating the physiological conditions of the membrane proteins environment. For instance, Muller et al. (12) showed that by changing the $\mathrm{pH}$ of the imaging solution, the purple membrane displayed conformational changes in its structure. Later studies demonstrated also that the application of forces induced changes in the bacteriorhodopsin structure (13), opening the way for "multidimensional" molecular imaging. In this regard, a more recent study of bacteriorhodopsin mechanical response (14) reached such a high level of precision that allowed the authors to determine the stiffest and the softest domains within a single protein. But the class of membrane proteins that is the most natural subject of investigation for a technique involving forces are the mechanosensitive proteins, e.g. PIEZO channels. In 
mechanosensitive proteins, the application of forces is thought to be responsible for key structural changes (like channel gating). This is exactly what a recent study searched for in a combination of cryo-EM and AFM experiments, where the authors were able to propose a mechanism that translates the forces applied to the membrane to channel gating (15).

What was lacking in these experiments was a dynamical point of view in the time-scale of seconds. The slow operation mode of conventional AFMs was boosted starting from the year 2010 when Toshio Ando and his group introduced the technical improvements described in section 2.2 and built the first HS-AFM, which could acquire several frames per second. The fast frame rate enabled the visualization of fast molecular mechanisms, for instance, the first direct imaging of the movements of myosin (16) and the diffusion of membrane proteins in membranes (17).

I believe that HS-AFM will become increasingly prominent for practical applications in drug discovery because it allows to directly probe the structural effects of ligands on the membrane protein of interest with a relatively simple machine (18) (Figure 4a and b). In the next section I will describe how AFM has been applied to real cell membranes.

\subsection{Imaging native cell membranes (from electron microscopy to $A F M$ )}

The question "how does a real cell membrane look like?" started to be answered only from the 1970s when the first rudimentary technique to break the cells and to create membrane-only samples was developed (19). Given the length-scale of the membrane features, the only microscopy technique available for their investigation was electron microscopy. This microscopy technique was extensively adopted in the following years, in particular thanks to a radical change that made the sample preparation more reliable: the use of sonic waves to break the cells to obtain the so called "unroofed" membranes (20). From these investigations we have learned how the membrane skeleton is arranged by looking directly into it from the cytoplasmic side of the membrane. For instance, it was possible to shed light on how the actin filament arrangements partition the plasma membrane, decreasing the lateral diffusion of lipids and proteins (21).

The versatility of AFM discussed also in the previous section comprises a relatively simpler instrument compared to the electron microscope and, more importantly, the possibility to work at room temperature with the sample under the physiological medium. Despite of these theoretical advantages on paper, the AFM was applied to native membranes relatively late, and starting with special compartments of the cell constituted just by membranes. An example of these applications is the rod outer segment disc, key for vision and rich in Rhodopsin (22). AFM was able to resolve the macromolecular structures of Rhodopsin in discs that form nano-domains with an average size of $500 \mathrm{~nm}^{2}(23)$.

Only more recently, a group in Japan revisited the unroofing technique developed for electron microscopy (24) a

Cryo-EM / Crystallography

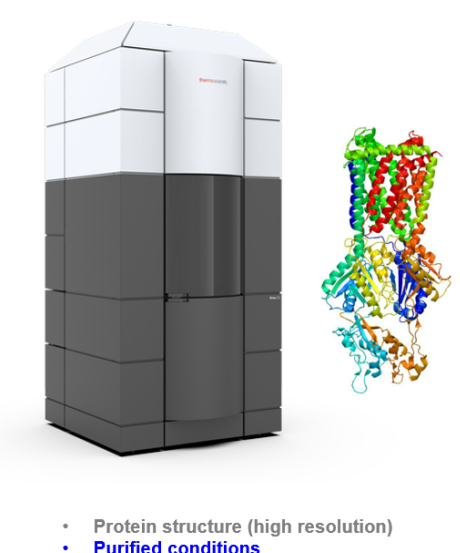

- Purified conditions b

AFM
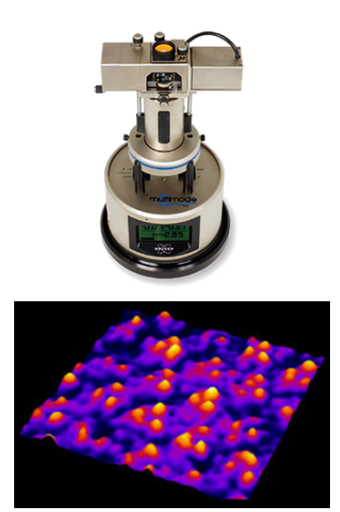

Protein conformations (low resolution) Protein dynamics Supramolecular structure in almost-physiological conditions

Cell and membrane mechanical properties

in vitro /ex vivo / purified conditions

\section{C}

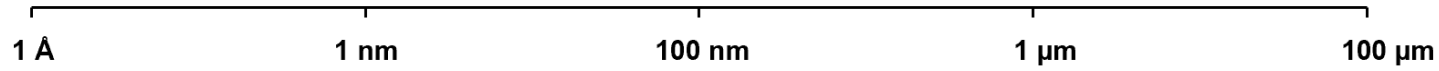

Figure 3. Comparison of imaging techniques for biomolecules and their domains of application. a: for structural, high-resolution, imaging. b: for intermediate molecular imaging with dynamics (AFM image obtained from JPK). c: for cellular imaging of biomolecules localization. 

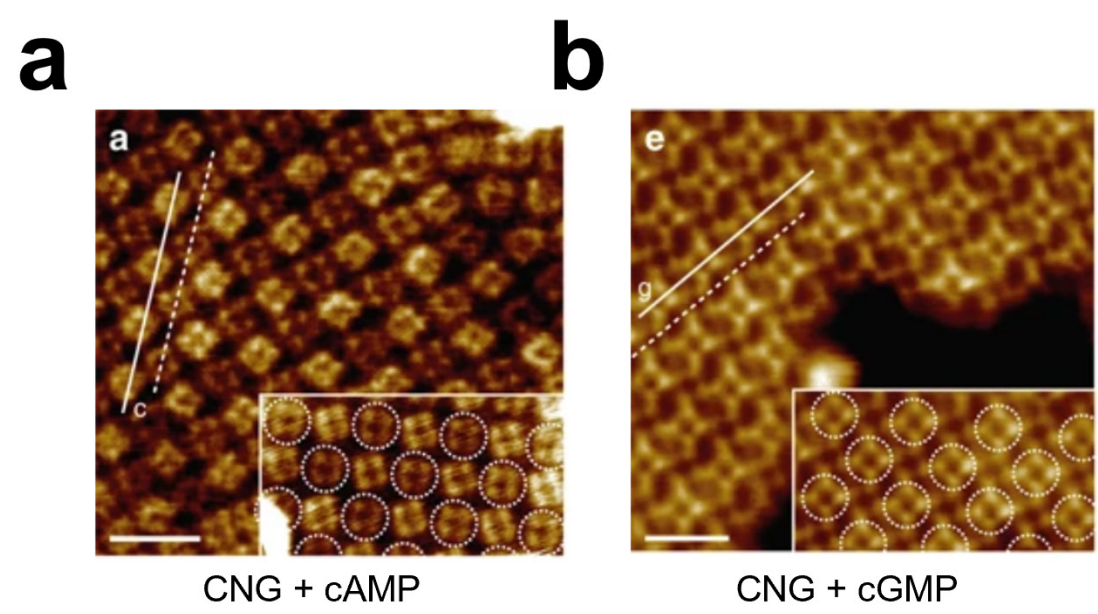

Figure 4. a and b: HS-AFM of conformational changes of an homolog of CNG channel in presence of cAMP and cGMP (adapted from (18)).

and imaged these samples after fixation with AFM, obtaining molecular resolution at room temperature (Figure 5a and b).

After having tested many of these methods, one of the major disadvantages of using AFM with native cell membranes is the fact that, the position of the isolated membrane itself is difficult to be found and requires time consuming scanning of the sample that also affects the quality of the tip. For this reason, inspired by an earlier study (25), I have developed an unroofing method that breaks only one single cell (or a few) on the very corner of a triangular coverslip (26) (Figure $\mathbf{5 c}$ and d). In this way, the identification of the position of the membrane is straightforward, and the membrane seems to be isolated without the membrane skeleton that was observed with other techniques. The very last goal in this case would be to image single membrane proteins with sub-molecular resolution in their complex macromolecular organization, potentially to recognize the signatures of diseases that affect directly the cell membrane.

\section{AFM as a force probe for cells and cell membranes}

In the previous sections I discussed the imaging capabilities of the AFM, but we cannot forget that the AFM is also an instrument able to measure forces at the micro- and nano-scale level. In the next sections I will present the major results of the past two decades obtained with the application of AFM-based force spectroscopy to biological materials.

\subsection{Single-cell force spectroscopy}

Cells are soft objects that range from 1 to 50 microns in size. As opposed to inanimate objects, cells are active entities constituted by a complex internal structure, they can duplicate and mix with other cells forming tissues, and finally organisms. The biochemical signals that the cells use to communicate and function have been the subject of molecular biology investigations which have made an enormous progress in the last 70 years. Different cell types are characterized by different biochemical signatures, and a question that one may ask is: are different cells characterized by different mechanical properties?

AFM can answer this question as the AFM tip can be used to indent a cell while recording the cantilever deflection. The recorded curve is informative of the cell stiffness and from the same curve it is possible to extrapolate conventional mechanical properties like the Young modulus (27). There is a vast scientific literature on this topic which focused very early on the attempt to find biomedical applications, in particular for cancer cell detection (28-30). The general conclusion of these studies is that cancer cells [lung, breast, pancreas (31), ovarian (29)] are softer and with a narrower standard deviation of the Young modulus compared to non-malignant cells of the same body cavity. Being softer may help cancer cells to be more dynamic and therefore successful in infiltrating tissues, but a definitive explanation of the problem is still to come.

More recently, people developed also some alternative operating mode to expand the range of mechanical properties that AFM can investigate. Yango el al. (32), for instance, introduced cycles of indentation and relaxation to measure both the Young modulus and the internal viscosity of the cell. An interesting advancement is the one introduced by Rigato et al. (33), where they measured the cell viscoelastic properties at different time scales, stimulating the cells from $1 \mathrm{~Hz}$ to $100 \mathrm{kHz}$. This is important because the cell is an active object whose constituents (e.g. cytoskeleton filaments) can rearrange at different time scales, therefore defining the 'mechanical fingerprint' can help to sort cells more accurately.

Being able to uniquely identify cancerous cells (or specimens) just by probing their mechanics, without the use of biochemical markers, seems to be a challenge that may be completed in relatively few years.

\subsection{Nanomechanics of lipid bilayers: from synthetic to native membranes}


The mechanical properties of the lipid bilayer - i.e. the component that separates the cell interior from the external environment - were not accessible with the standard biochemical techniques available until the invention of the AFM. AFM opened the way for the direct investigation of the strength of molecular interactions among lipids in supported membranes. In these experiments, synthetic lipids (e.g. dipalmitoylphosphatidylcholine and dioleoylphosphatidylcholine) are mixed in solution and deposited on atomically flat surfaces (e.g. cleaved mica), then they are imaged with AFM to confirm their correct assembly, and finally indented with the AFM tip to probe the nanomechanical properties of the membrane. The prototypical indentation curve recorded by the AFM shows an initial elastic behavior of the membrane (deformation of the bilayer), until the compression force of the AFM tip reaches a critical value that causes the breakage of the bilayer. After the breakage, the tip is retracted while the membrane self-assembles back, closing the hole.

To my knowledge, the first experiments on lipids nanomechanics were performed in 1999 by Dufrene et al. (34): they demonstrated the versatility of AFM to probe the chemical and physical properties of lipid membranes. After these pioneering studies, many labs around the world started to apply AFM to membranes, characterizing their behavior in many different conditions of physiological relevance. For instance, it was shown that the stiffness of the membrane increases by reducing its temperature (35) or by increasing the percentage of cholesterol (36), with important consequences for our understanding of the dynamic of processes at cellular and sub-cellular levels. It is surprising how, from relatively crude indentations and enough statistics, it is possible to extrapolate very detailed physical quantities of the bilayer like the line tension and the spreading pressure, quantities that are essential in coarse grain simulations of the cell.
I would like to mention two noteworthy recent experimental advancements in the field of membrane biophysics. Goncalves et al. (37), instead of studying supported membranes, developed a substrate with submicrometric holes where the membrane could remain free-standing, emulating a real cell surface. In this way, they were able to precisely measure the breaking point of a membrane that could bend under the AFM tip push. While Al-Rekabi et al. (38), similarly to Rigato et al. (33) as described in the previous section, introduced a multifrequency AFM mode that allows to capture the viscoelastic properties of the membrane and demonstrates how cholesterol concentration is a key ingredient in changing membrane properties from viscous to elastic.

The goal of the recently developed single-cell unroofing (26) is to transfer the application of the powerful methods described above (and benchmarked with synthetic membranes) to native cellular membranes, in order to gather information of the real nanomechanics generated by the native complexity. Indeed, with single-cell unroofing it is possible to tear the cell membrane of cells (Figure 5c) and directly probe their indentation force, deriving elasticity and viscosity of cells of patients.

\section{AFM as a force probe for single proteins}

Measuring the mechanical properties of cells or cell membranes is definitely a technological leap, but it does not represent a conceptual breakthrough, in the sense that these results could be somehow expected. What really represents a practical revolution for an unsolved problem in biology is the possibility to record the unfolding of single proteins - one at a time - with AFM-based singlemolecule force spectroscopy (SMFS).

In fact, the physico-chemical process that guides a linear sequence of amino acids into the three-dimensional precise structures that (almost) every protein shows may a
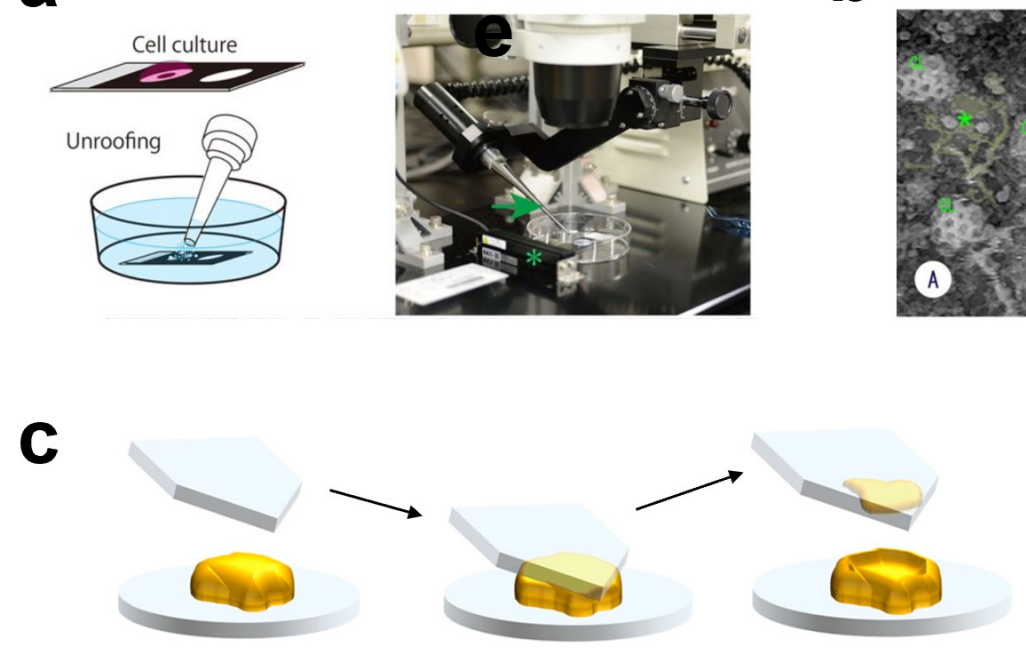

b

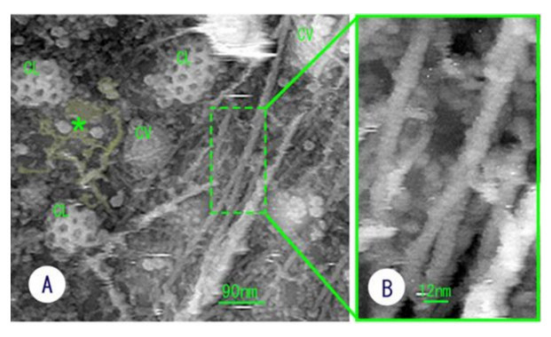

d

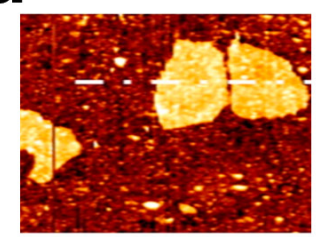

Figure 5. a \& b: unroofing preparation through sonic waves and AFM imaging of actin filaments and clathrin (24). c \& d: single cell unroofing through the gentle squeezing of a single cell and the resulting isolated membrane like (26). 

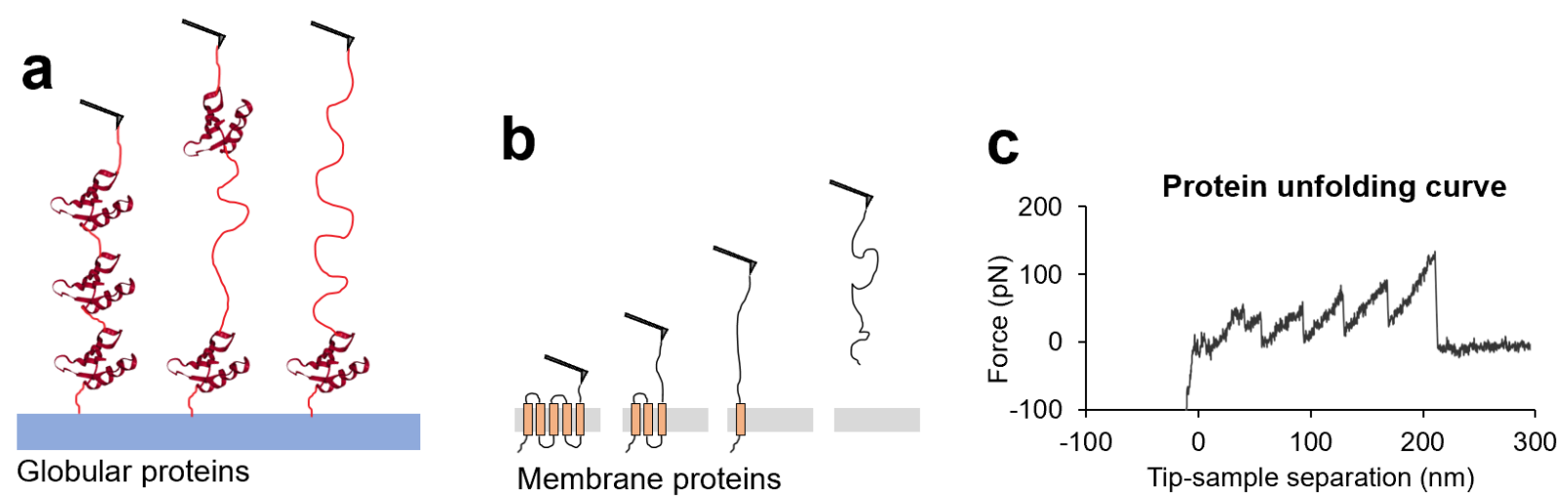

Figure 6. a: unfolding of a tandem of globular protein. b: unfolding of a trans-membrane protein. c: prototypical unfolding curve od a protein.

be taken for granted, but it has been a puzzle for physicists since the 1950s (39). The number of variables that should be taken into account to simulate with atomic resolution the folding of a protein is simply intractable for modern computational power, and therefore, in the past years many computational "shortcuts" have been invented to tackle the problem in an approximate manner (40). On the other hand, from the experimental point of view, the only way to study protein folding and unfolding was by chemical denaturation (41), thus at the ensemble level and not at the single-molecule level. There was no certain mechanistic understanding of how the dynamics of protein folding should work.

This is the context where SMFS, starting from the late $1990 \mathrm{~s}$, revolutionized the way people study protein folding. The experimental setup consists in an AFM cantilever tip that, instead of being used for imaging as discussed in section 3.1, is approached and retracted form a flat sample onto which many copies of the same protein of interest are deposited. The AFM tip pushes the sample and binds one of the terminus ends of the underlying protein. Then the tip is retracted while recording the force experienced by the cantilever, so that it is possible to form a force vs distance spectrum (F-D curve). If the other terminus of the protein is bounded to the sample surface, the tip will literally stretch the protein, forcing denaturation. The prototypical unfolding curve of a sequence of immunoglobulin domains was observed for the first time by Rief et al. in 1997 (42) (Figure 6a) showing the so called saw-tooth like shape, i.e. a sequence of rising phases followed by vertical jumps to the baseline at zero-force.

How can we describe this behavior? The rising phases can be fitted with the worm-like chain model, i.e. a model developed in polymer physics that describes the stretching of an ideal semi-flexible chain (43). The sudden jump to zero-force is instead representative of the abrupt unfolding of a complete protein domain, which is unexpected. The unfolding of the protein does not happen in a continuous way, but rather in an all-or-none fashion. This two-state behavior (folded vs unfolded) is typical for the majority of globular proteins investigated so far, e.g. GFP, Xylanase, Fibronectin and many others (44). Moreover, these globular proteins - when unfolded - tends to fold back into their original 3D structure even in vitro (42).

Membrane proteins, and in particular trans-membrane proteins, are topologically very different from globular proteins because, instead of being fully immersed in the cytosol, they perform their function embedded in the cell membrane. They are also more difficult to be studied since they are laborious to be purified and they don't crystallize well (it is often necessary to study homologous proteins that are easier to be handled). The fact that they operate across the membrane introduces an additional complex ingredient to the problem of folding, indeed it was discovered that trans-membrane proteins need the help of other proteins (translocon) to be correctly folded into the membrane (45).

SMFS has what it takes to be very powerful when applied to membrane proteins, possibly being informative also of membrane protein structure. The first experimental observation dates back to the year 2000 when Oesterhelt et al. (46) used AFM-based SMFS to unfold bacteriorhodopsin. The unfolding curve of bacteriorhodopsin (see Figure $6 \mathbf{b}$ and c) resembles the unfolding of a tandem of globular proteins (Figure 6a) but it has a slightly different interpretation. As opposed to what happens to the majority of globular proteins that unfold in an all-or-none fashion, in bacteriorhodopsin the different domains of the same molecule unfold separately and consecutively, one after the other. A general empirical observation is that these domains correspond to pairs of alpha helices (or beta hairpin). This observation is actually quite general, since further studies of other membrane proteins tend to support this empirical "rule", also in betabarrel membrane proteins. E.g. aquaporin (47), sodiumproton antiporter (48), FhuA (49), etc. The unfolding behavior of membrane proteins is somehow reminiscent of the mechanistic intuition of how these proteins should exit the membrane when pulled.

However, thanks to a recent improvement in resolution of the AFM probes, it has been shown that the single and 


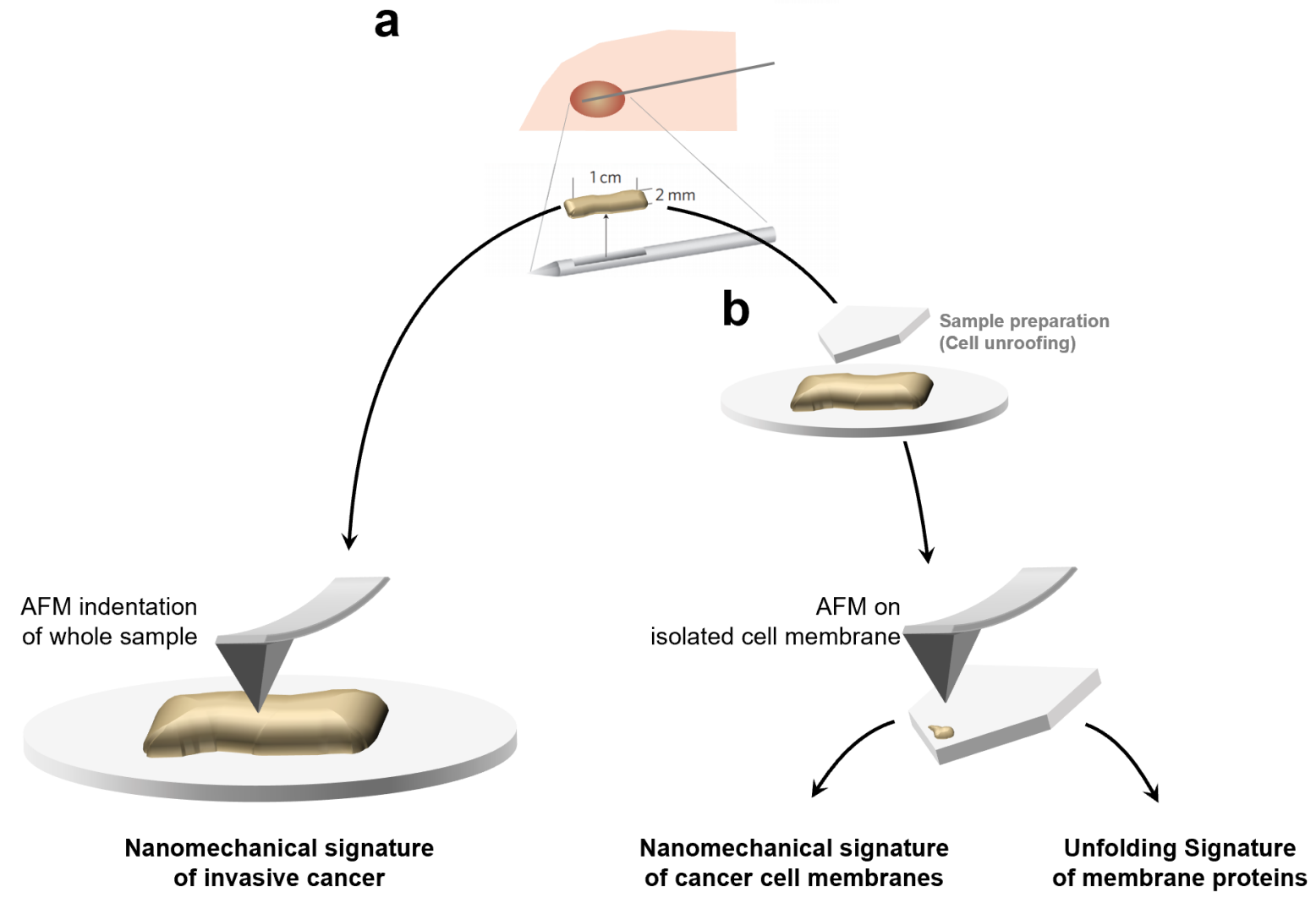

Figure 7. a: schematic of a biopsy of a patient. b: sample preparation: cell membrane isolation from biopsy cells. AFM force measurements performed on the biopsy or on the cell membranes reveal signatures that are characteristic of cancerous samples.

separated peaks of unfolding that was reported in the past, that correspond to the unfolding of pairs of alpha helices, are actually decorated by a rich series of minor unfolding events, indicating that the complexity of the process is still highly unresolved (50).

But with SMFS it is possible to test not only the detailed physics of folding, but also to obtain biological insights on the structure-function of specific proteins. An example is the work by Serdiuk et al., where they directly observed the action of chaperon proteins acting on membrane proteins that were previously unfolded, showing that without the help of these chaperons, a membrane protein cannot autonomously fold back into the membrane (51).

In the previous paragraphs I described how SMFS can be used to explore the problem of protein folding, but I strongly believe that the major applications of SMFS are to problems at the intersection with biomedicine. SMFS can be exploited to investigate protein behaviors where other techniques cannot be applied. I will now report some applications in this direction. One way to use SMFS to tackle a biomedical problem is to study the differences of a membrane protein and a mutated one that may be involved in a disease. Kawamura et al. (52) took a similar direction studying the differences between rhodopsin and opsin, which is the same protein not bounded to the chromophore that enables vision. In this case they detected slightly differences in the unfolding behavior, showing that SMFS can resolve these changes. Another approach is to study the mechanical differences induced by the binding of a ligand to a membrane protein, and how it affects the structure (53).

What seems a highly unexplored area of research is the one touched by Otten et al. (44), i.e. trying to change the scale and the throughput of the SMFS experiments. They developed an interesting platform that can express proteins in vitro, and then screen them in order to create a SMFS resource to test the most various hypothesis related to protein mechanics or folding, for instance malfunction due to misfolding.

\section{Future perspectives}

Much of the research presented in this review deals with new knowledge generated by AFM applied to biological samples. This knowledge has direct implications in real life problems.

It is estimated that the amount of funding that has been spent in basic and applied research involving bio-AFM is in the order of some billion dollars. Given the tremendous collaborative efforts of the last 30 years, it is reasonable to believe that a certain amount of organizations will eventually succeed in commercializing an AFM-based device, sold to hospitals. To my knowledge, the practical implications that nowadays are at the proof-of-concept level, and that could help the diagnosis of diseases are the 
ones depicted in Figure 7:

i. direct indentation measurements of biopsies for automated tissue diagnostics (30) (technology readiness level 7-8, e.g. ARDITIS AG (Swiss company));

ii. indentation measurements of the cell membranes isolated from biopsies (26) (technology readiness level $3-4)$;

iii. single-molecule force spectroscopy the native cell membranes isolated from biopsies (54) (technology readiness level 3-4).

The direct indentation of biopsies and the correlation of these data with the state of the patient is with no doubt the technology closest to a commercial device. This tool seems to help the physicians to identify the malignancy of different cancer biopsies from patients. There are clear substantial advancements also in hybrid systems of AFM plus Raman spectroscopy (55). In these cases, the benefit manifests in terms of chemical recognition of the material with sub-micrometer resolution; but these techniques are at the moment still difficult to be operated and still unmatured, but with high potential.

It is improbable that only one of these techniques will revolutionize the diagnostics by its own, but it is most likely that the intercombination of the mechanical signatures of each of these approaches, together with parallel diagnostic tools, will substantially help the decisions of physicians, even in a near future.

\section{Conflict of interest}

The authors declare that they have no conflict of interest.

\section{References}

1. Binnig G, Quate CF, Gerber Ch. Atomic force microscope. Phys Rev Lett. 1986 Mar 3;56(9):930-3.

2. McClelland GM, Erlandsson R, Chiang S. Atomic force microscopy: general principles and a new implementation. In: Thompson D.O., Chimenti D.E. (eds) Review of Progress in Quantitative Nondestructive Evaluation. 1987, vol 6 A Springer, Boston, MA

3. Chen I-C, Chen L-H, Ye X-R, Daraio C, Jin S, Orme CA et al. Extremely sharp carbon nanocone probes for atomic force microscopy imaging. Appl Phys Lett. 2006 Apr 10;88(15):153102.

4. Edwards DT, Faulk JK, LeBlanc M-A, Perkins TT. Force spectroscopy with 9- $\mu$ s resolution and sub-pN stability by tailoring AFM cantilever geometry. Biophys J. 2017 Dec 19;113(12):2595-600.

5. Ando T, Uchihashi T, Kodera N. High-speed AFM and applications to biomolecular systems. Annu Rev Biophys. 2013;42(1):393-414.

6. Kobayashi H, Ogawa M, Alford R, Choyke PL, Urano Y. New strategies for fluorescent probe design in medical diagnostic imaging. Chem Rev. 2010 May 12;110(5):2620-40.

7. Yguerabide J, Yguerabide EE. Fluorescence spectroscopy in biological and medical research. Int J Radiat Appl Instrum C Radiat Phys Chem. 1988 Jan 1;32(3):457-64.

8. Liao M, Cao E, Julius D, Cheng Y. Single particle electron cryo-microscopy of a mammalian ion channel. Curr Opin Struct Biol. 2014 Aug 1;27:1-7.

9. Williams $\mathrm{DH}$, Mitchell T. Latest developments in crystallography and structure-based design of protein kinase inhibitors as drug candidates. Curr Opin Pharmacol. 2002 Oct 1;2(5):567-73.
10. Yin $H$, Flynn AD. Drugging membrane protein interactions. Annu Rev Biomed Eng. 2016;18(1):51-76.

11. Worcester DL, Miller RG, Bryant PJ. Atomic force microscopy of purple membranes. J Microsc.1988;152(3):817-21.

12. Müller DJ, Schabert FA, Büldt G, Engel A. Imaging purple membranes in aqueous solutions at sub-nanometer resolution by atomic force microscopy. Biophys J.1995 May 1;68(5):1681-6.

13. Müller DJ, Büldt G, Engel A. Force-induced conformational change of bacteriorhodopsin. J Mol Biol.1995 Jan 1;249(2):239-43.

14. Rico F, Su C, Scheuring S. Mechanical mapping of single membrane proteins at submolecular resolution. Nano Lett. 2011 Sep 14;11(9):3983-6.

15. Lin Y-C, Guo YR, Miyagi A, Levring J, Mackinnon R Scheuring S. Force-induced conformational changes in PIEZO1. Nature. 2019 Sep;573(7773):230-4.

16. Kodera N, Yamamoto D, Ishikawa R, Ando T. Video imaging of walking myosin $\mathrm{V}$ by high-speed atomic force microscopy. Nature. 2010 Nov;468(7320):72-6.

17. Casuso I, Khao J, Chami M, Paul-Gilloteaux P, Husain $M$, Duneau J-P, et al. Characterization of the motion of membrane proteins using high-speed atomic force microscopy. Nat Nanotechnol. 2012 Aug;7(8):525-9.

18. Marchesi A, Gao X, Adaixo R, Rheinberger J, Stahlberg H Nimigean $C$, et al. An iris diaphragm mechanism to gate a cyclic nucleotide-gated ion channel. Nat Commun. 2018 Sep 28;9(1):1-11.

19. Clarke M, Schatten G, Mazia D, Spudich JA. Visualization of actin fibers associated with the cell membrane in amoebae of Dictyostelium discoideum. PNAS. 1975 May 1;72(5):1758-62.

20. Heuser J. The Production of 'cell cortices' for light and electron microscopy. Traffic. 2000 Jul 1;1(7):545-52.

21. Morone N, Fujiwara T, Murase K, Kasai RS, Ike H, Yuasa $\mathrm{S}$, et al. Three-dimensional reconstruction of the membrane skeleton at the plasma membrane interface by electron tomography. J Cell Biol. 2006 Sep 11;174(6):851-62.

22. Tanuj Sapra K, Park PS-H, Filipek S, Engel A, Müller DJ, Palczewski K. Detecting molecular interactions that stabilize native bovine rhodopsin. J Mol Biol. 2006 Apr;358(1):255-69.

23. Whited AM, Park PS-H. Nanodomain organization of rhodopsin in native human and murine rod outer segment disc membranes. Biochim Biophys Acta Biomembr. 2015 Jan 1;1848(1, Part A):26-34.

24. Usukura E, Narita A, Yagi A, Ito S, Usukura J. An unroofing method to observe the cytoskeleton directly at molecular resolution using atomic force microscopy. Sci Rep. 2016 Jun 7;6:srep27472

25. Nermut MV, Williams LD. Freeze-fracturing of monolayers (capillary layers) of cells, membranes and viruses: some technical considerations. J Microsc. 1977 Jul 1;110(2):121-32.

26. Galvanetto N. Single-cell unroofing: probing topology and nanomechanics of native membranes. Biochim Biophys Acta Biomembr. 2018 Dec;1860(12):2532-8.

27. Faria EC, Ma N, Gazi E, Gardner P, Brown M, Clarke NW, et al. Measurement of elastic properties of prostate cancer cells using AFM. Analyst. 2008 Oct 20;133(11):1498-500.

28. Lulevich V, Zink T, Chen H-Y, Liu F-T, Liu G. Cell mechanics using atomic force microscopy-based single-cell compression. Langmuir. 2006 Sep 1;22(19):8151-5.

29. Xu W, Mezencev R, Kim B, Wang L, McDonald J, Sulchek T. Cell stiffness is a biomarker of the metastatic potential of ovarian cancer cells. PLoS One. 2012 Oct;7(10):e46609.

30. Plodinec M, Loparic M, Monnier CA, Obermann EC, ZanettiDallenbach $\mathrm{R}$, Oertle $\mathrm{P}$, et al. The nanomechanical signature of breast cancer. Nat Nanotechnol. 2012 Nov;7(11):757-65.

31. Cross SE, Jin Y-S, Rao J, Gimzewski JK. Nanomechanical analysis of cells from cancer patients. Nat Nanotechnol. 2007 Dec;2(12):780-3. 
32. Yango A, Schäpe J, Rianna C, Doschke H, Radmacher M Measuring the viscoelastic creep of soft samples by step response AFM. Soft Matter. 2016 Oct 12;12(40):8297-306.

33. Rigato A, Miyagi A, Scheuring S, Rico F. High-frequency microrheology reveals cytoskeleton dynamics in living cells. Nat Phys. 2017 Aug;13(8):771-5.

34. Dufrêne YF, Boland T, Schneider JW, Barger WR, Lee GU. Characterization of the physical properties of mode biomembranes at the nanometer scale with the atomic force microscope. Faraday Discuss. 1999;111(0):79-94.

35. Leonenko ZV, Finot E, Ma H, Dahms TES, Cramb DT. Investigation of temperature-induced phase transitions in DOPC and DPPC phospholipid bilayers using temperaturecontrolled scanning force microscopy. Biophysical Journal. 2004 Jun 1;86(6):3783-93

36. Sullan RMA, Li JK, Hao C, Walker GC, Zou S. CholesterolDependent Nanomechanical Stability of Phase-Segregated Multicomponent Lipid Bilayers. Biophys J. $2010 \mathrm{Ju}$ 21;99(2):507-16.

37. Gonçalves RP, Agnus G, Sens P, Houssin C, Bartenlian B, Scheuring S. Two-chamber AFM: probing membrane proteins separating two aqueous compartments. Nat Methods. 2006 Oct 22;3(12):nmeth965.

38. AI-Rekabi Z, Contera S. Multifrequency AFM reveals lipid membrane mechanical properties and the effect of cholesterol in modulating viscoelasticity. PNAS. $2018 \mathrm{Mar}$ 13;115(11):2658-63.

39. Anfinsen CB, Redfield RR, Choate WL, Page J, Carroll WR Studies on the gross structure, cross-linkages, and termina sequences in ribonuclease. J Biol Chem. 1954;207(1):201-10.

40. Laio A, Parrinello M. Escaping free-energy minima. PNAS 2002 Oct 1;99(20):12562-6.

41. Fong S, Hamill SJ, Proctor M, Freund SMV, Benian GM, Chothia $\mathrm{C}$, et al. Structure and stability of an immunoglobulin superfamily domain from twitchin, a muscle protein of the nematode Caenorhabditis elegans. J Mol Biol. 1996 Dec 6;264(3):624-39.

42. Rief M, Gautel M, Oesterhelt F, Fernandez JM, Gaub HE Reversible unfolding of individual titin immunoglobulin domains by AFM. Science. 1997;276(5315):1109-1112.

43. Doi M, Edwards SF. The theory of polymer dynamics. Clarendon Press; 1988. 420 p.
44. Otten M, Ott W, Jobst MA, Milles LF, Verdorfer T, Pippig DA, et al. From genes to protein mechanics on a chip. Nat Methods. 2014 Nov;11(11):1127-30.

45. Johnson AE, van Waes MA. The translocon: a dynamic gateway at the ER membrane. Annu Rev Cell Dev Biol. 1999;15:799-842.

46. Oesterhelt F, Oesterhelt D, Pfeiffer M, Engel A, Gaub HE, Müller DJ. Unfolding pathways of individual bacteriorhodopsins. Science. 2000 Apr 7;288(5463):143-6.

47. Möller C, Fotiadis D, Suda K, Engel A, Kessler M, Müller DJ. Determining molecular forces that stabilize human aquaporin-1. J Struct Biol. 2003 Jun 1;142(3):369-78.

48. Kedrov A, Ziegler C, Janovjak H, Kühlbrandt W, Müller DJ. Controlled unfolding and refolding of a single sodium-proton antiporter using atomic force microscopy. J Mol Biol. 2004 Jul 23;340(5):1143-52.

49. Thoma J, Bosshart P, Pfreundschuh M, Müller DJ. Out but not in: the large transmembrane $\beta$-barrel protein FhuA unfolds but cannot refold via $\beta$-hairpins. Structure. $2012 \mathrm{Dec}$ 5;20(12):2185-90.

50. Yu H, Siewny MGW, Edwards DT, Sanders AW, Perkins TT. Hidden dynamics in the unfolding of individual bacteriorhodopsin proteins. Science. $2017 \mathrm{Mar}$ 3;355(6328):945-50.

51. Serdiuk T, Balasubramaniam D, Sugihara J, Mari SA, Kaback HR, Müller DJ. YidC assists the stepwise and stochastic folding of membrane proteins. Nat Chem Biol. 2016 Nov;12(11):911-7.

52. Kawamura S, Gerstung M, Colozo AT, Helenius J, Maeda A, Beerenwinkel N, et al. Kinetic, energetic, and mechanical differences between dark-state rhodopsin and opsin. Structure. 2013 Mar 5;21(3):426-37.

53. Spoerri PM, Sapra KT, Zhang C, Mari SA, Kato HE, Kobilka $\mathrm{BK}$, et al. Conformational plasticity of human proteaseactivated receptor 1 upon antagonist- and agonist-binding. Structure. 2019 Oct;27(10):1517-1526.

54. Galvanetto N, Maity S, Ilieva N, Ye Z, Laio A, Torre V. Unfolding and identification of membrane proteins from native cell membranes. bioRxiv. 2019 Sep;26;732933.

55. Fu W, Zhang W. Hybrid AFM for nanoscale physicochemical characterization: recent development and emerging applications.Small. 2017, 425;1603525. 